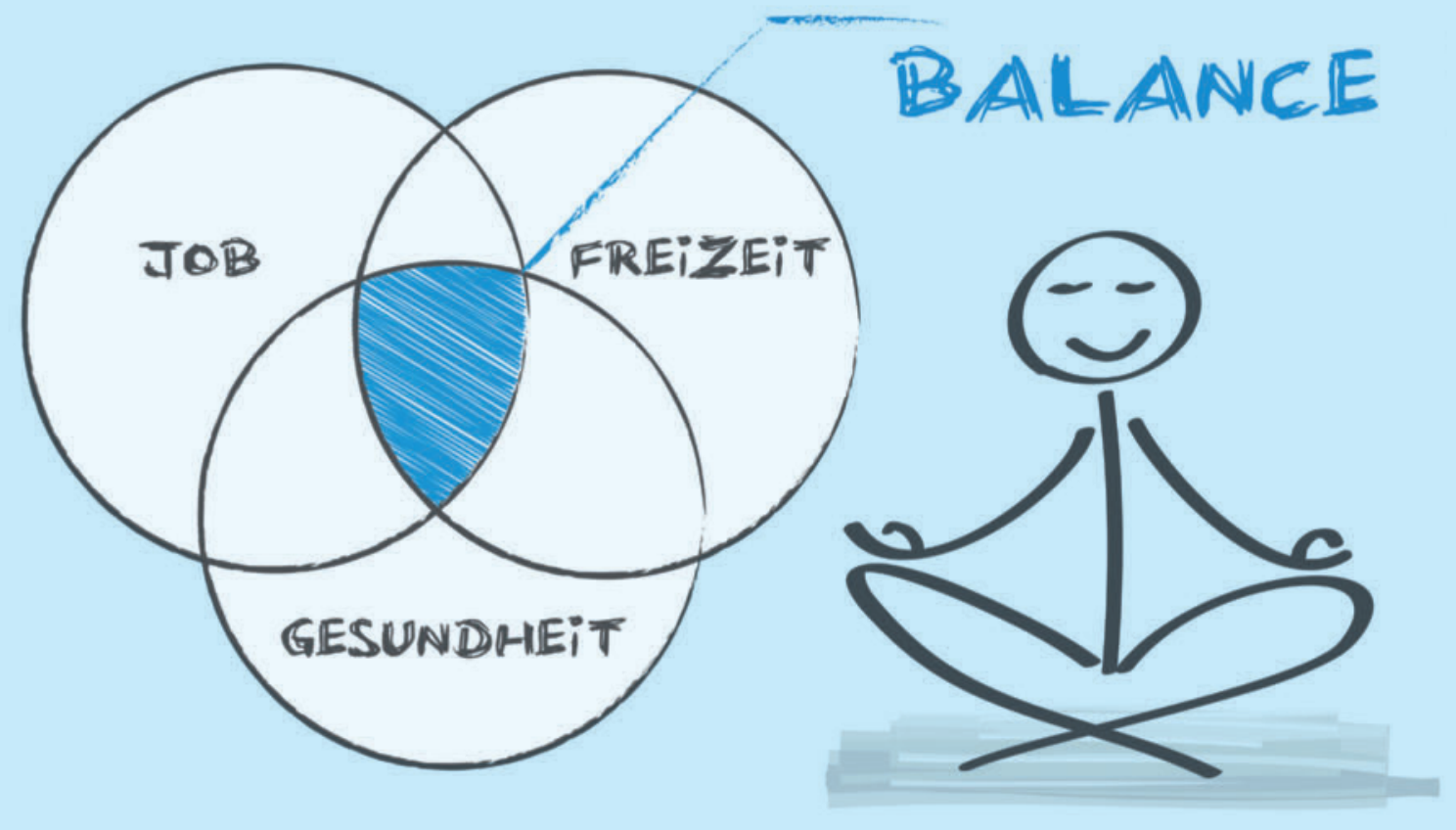

Wenn die Arbeitsbelastung steigt, kommt die Occupational Balance ins Wanken - insbesondere in Zeiten der Pandemie und in Gesundheitsberufen. Quelle: () Trueffelpix/stock.adobe.com

\title{
Gesundheitsberufler aus dem Gleichgewicht
}

Occupational Balance in der Pandemie

$\rightarrow$ Während der Covid-19-Pandemie ist die Occupational Balance von Angehörigen der Gesundheitsberufe erheblich beeinträchtigt. Dies betrifft vor allem das Gleichgewicht zwischen Produktivität und Freizeit. Aktivitäten der Selbstversorgung sind von der Dysbalance weniger stark betroffen. Zu diesem Ergebnis kommt ein Team um Güleser Güney Yılmaz von der Kütahya Health Sciences University, Türkei.

Die Forschenden führten die Studie im Mixed Methods Design mit 206 Gesundheitsberuflern wie Ergo- und Physiotherapeut*innen oder Pflegenden durch. Die durchschnittlich 31-jährigen und überwiegend weiblichen Teilnehmenden wurden in eine Studien- und eine Kontrollgruppe unterteilt. Die Studiengruppe $(n=105)$ hatte direkten Kontakt zu an Corona erkrankten Menschen. Die Kontrollgruppe $(n=101)$ arbeitete nicht direkt mit Erkrankten.

Zunächst erhob die Forschungsgruppe quantitative Daten. Dazu befragte sie alle Teilnehmenden mit dem türkischen Fragebogen zur Occupational Balance (OBQ11-T). Dieser misst die Zufriedenheit mit Betätigungen anhand des Umfangs und deren Vielfalt. Im Anschluss daran führten die Forschenden halbstrukturierte Interviews mit den Teilnehmenden durch. Darin fragten sie unter anderem nach Alltagsroutinen, neuen Aktivitäten oder Gründen für eine unterbrochene Occupational Balance.
Die Ergebnisse des Assessments zur Occupational Balance zeigen, dass sich die Studiengruppe in einem größeren Ungleichgewicht befand als die Kontrollgruppe. Diese Dysbalance wurde verstärkt, wenn die Teilnehmenden selbst an Covid-19 erkrankt waren. Aus den Interviews ging hervor, dass sich bei 36 Prozent der Befragten die persönlichen Aktivitäten verändert hatten, zum Beispiel durch Schlafstörungen. Bei etwa 41 Prozent hatte sich die Occupational Balance durch die erhöhte Arbeitsbelastung verschlechtert. Mehr als die Hälfte nannte negative Auswirkungen der Pandemie auf Freizeit und Erholung. So gaben sie an, dass sie ihre Familien nicht sehen konnten oder aufgehört hatten, Sport zu treiben. 57 Prozent passten ihre Betätigungen an oder vermieden sie, da sie fürchteten, Corona auf andere zu übertragen. Des Weiteren gaben die Befragten an, dass ihre Occupational Balance durch die Mehrarbeit und die belastenden Hygienemaßnahmen negativ beeinflusst wurde.

Die Forschenden schlussfolgern, dass Angehörige der Gesundheitsberufe aufgrund von Müdigkeit, psychischer Erschöpfung und Zeitmangel ihrer Freizeit nicht angemessen nachgehen konnten. Sie empfehlen, die Occupational Balance insbesondere während der Pandemie im Hinblick auf soziale Partizipation, Zeitnutzung und Selbstfürsorge zu beachten. ms Aust Occup Ther J 2021; 68: 520-534 


\section{Selbstständigkeitstraining und Sturzprophylaxe am effektivsten}

\begin{abstract}
Gemeindenahe Ergotherapie $\rightarrow$ Trotz zunehmender Bedeutung lässt sich die Evidenz gemeindenaher ergotherapeutischer Behandlungsansätze nur spärlich nachweisen. MariaFrancesca Estrany-Munar der ASPAYM Baleares, Spanien, und ihr Team gingen in ihrer Übersichtsarbeit der Frage nach, wie umfangreich die Forschung zu gemeindenaher Ergotherapie ist und ob sich Evidenzlevel zur Wirksamkeit der Interventionen ableiten lassen.
\end{abstract}

Sie durchsuchten sechs Datenbanken und wählten 122 Forschungsarbeiten mit qualitativem und quantitativem Design für die weiteren Analyseschritte aus. Nach Überprüfung der wissenschaftlichen Qualität filterten sie zwölf quantitative Studien für die finale Statistikauswertung aus. In diesem Zuge überprüften sie, inwieweit sich aus den Forschungsinhalten
Rückschlüsse für das gesamte Feld der gemeindenahen Ergotherapie schließen lassen.

Die Ergebnisse zeigen, dass sich ergotherapeutische Angebote schwerpunktmäßig mit älteren und psychisch erkrankten Menschen in der Gemeinde beschäftigen. Im geriatrischen Bereich zielen die Interventionen vor allem auf den Erhalt der selbstständigen Alltagsbewältigung, auf Sturzprophylaxe und ein verbessertes Wohlbefinden ab. Angebote für psychisch erkrankte Menschen enthielten hingegen Strategien zur Verbesserung der sozialen Teilhabe, zur Stimmungsstabilisierung und Rückfallprophylaxe bei Abhängigkeitserkrankungen.

Während sich aus den Studien keine Aussagen zur Wirksamkeit der Interventionen bei psychisch erkrankten Gemeindemitgliedern ableiten lassen, sind für Ältere die Angebote zur Vermeidung von Stürzen und zum Erhalt der Kompetenzen im Bereich ADL am effektivsten. Die Interventionen erreichen jedoch nur mittlere bis niedrige Evidenzlevel.

Die Forschungsgruppe hält eine einheitliche Definition für gemeindenahe Ergotherapie für nötig, um die Wirksamkeit und Expertise in diesem Bereich besser abbilden zu können. Ausgehend vom weit gefassten Gesundheitsbegriff gewinnen zunehmend ergotherapeutische Interventionen an Bedeutung, die sich nicht nur auf das Individuum, sondern auf die gesamte Gemeinde konzentrieren. Weitere Forschung sollte sich auf Gesundheitsförderung und Prävention konzentrieren, da es bei diesen Themen bislang zu wenig hochwertige Studien gibt, um die Evidenz nachweisen zu können. rehe Int J Environ Res Public Health 2021; 18: 3142 


\section{Kein Mehrwert durch zusätzliches Eltern-Coaching}

$\mathrm{CO}-\mathrm{OP} \rightarrow$ Die Intervention „Cognitive Orientation to daily Occupational Performance“ (CO-OP) kann bei Kindern mit einer entwicklungsbezogenen Koordinationsstörung (Developmental Coordination Disorder, DCD) die Betätigungsperformanz verbessern. Ein zusätzliches Elterncoaching mittels Occupational Performance Coaching ( $\odot \mathrm{OPC}$ ) bringt jedoch keine weitere Verbesserung. Zu diesem Ergebnis kommen Prof. Clarice Ribeiro Soares Araujo an der Federal University of Paraíba in João Pessoa, Brasilien, und ihr Team in einer randomisierten klinischen Studie.

Über verschiedene Wege wie Wartelisten für Kinder, die mit Verdacht auf eine Entwicklungsstörung zur Ergotherapie überwiesen wurden, Einladungen an Grundschulen und Anzeigen in sozia-

len Medien rekrutierte die Forschungsgruppe 22 Kinder. Die 4 Mädchen und 18 Jungen waren durchschnittlich 8,96 Jahre alt und wurdern per Zufall der Interventions- oder Kontrollgruppe zugeordnet. Verschiedene Assessments kamen zu Beginn und nach der Intervention zum Einsatz, darunter COPM, PEM-CY, MABC-2, FDT und TOL. Die Kinder beider Gruppen erhielten zwölf 60-minütige CO-OP-Sitzungen ein bis zweimal pro Woche. Die Eltern der Interventionsgruppe nahmen zusätzlich zu den drei im CO-OP vorgesehenen

Sitzungen an fünf weiteren Gruppensitzungen teil. Sie wurden in kollaborative und zielgerichtete Gespräche eingebunden, um Faktoren und Hindernisse zu ermitteln, die die Betätigungsperformanz ihrer Kinder beeinflussen.

Beide Gruppen zeigten am Ende des Untersuchungszeitraums vergleichbare Verbesserungen in der Betätigungsperformanz und bei den motorischen Leistungen. Es gab keine signifikanten Unterschiede durch die zusätzlichen OPC-Sitzungen. Die Interventionsgruppe war jedoch zufriedener mit den Verbesserungen.

95

\section{Eltern benötigen ange- messene Informationen und Unterstützung.}

Dies könnte mit der veränderten Wahrnehmung der Eltern für die Betätigungsprobleme ihrer Kinder zusammenhängen. Darüber hinaus zeigte sich, dass Strategien die Mitarbeit der Eltern verbesserten: der regelmä-

Bige Kontakt mit den Familien, der Austausch von Ideen für Hausaufgaben, die ständige Erinnerung an das Heft und daran, wie es helfen kann, Strategien in anderen Kontexten auszuprobieren, sowie Videos und Botschaften zur Erledigung der Hausaufgaben.

Ein zusätzliches Coaching ist möglicherweise nicht notwendig, wenn die Eltern angemessene Informationen und Unterstützung erhalten, während sie an der betätigungs- und klientenzentrierten Therapie ihrer Kinder teilhaben. kj Res Dev Disabil 2021; 110: 103862 $\rightarrow$ Glossar

\section{Assessments \\ COPM}

Das Canadian Occupational Performance Measure misst die subjektiv wahrgenommene Qualität der Betätigungsperformanz und die Zufriedenheit damit mittels 10-Punkte-Skala.

\section{PEM-CY}

Im Participation and Environment Measure for Children and Youth werden Eltern zur Partizipation ihrer 5- bis 7-jährigen Kinder befragt. Das Assessment misst die Häufigkeit der Teilnahme, den Grad der Beteiligung und den Wunsch nach Veränderung bei 25 Aktivitäten zu Hause, in der Schule und Gemeinde.

\section{MABC-2}

Der motorische Leistungstest besteht aus acht Aufgaben zu manueller Geschicklichkeit, Zielen und Fangen sowie Gleichgewicht.

\section{FDT}

Der Fünf-Ziffern-Test misst die kognitive Flexibilität und die hemmende Kontrolle. Er besteht aus vier Teilen (Lesen, Zählen, Auswählen, Umschalten) und eignet sich für Personen unterschiedlichen Alters, Bildungsstandes und kulturellen Hintergrunds. Voraussetzung sind minimale Sprachkenntnisse.

TOL

Der Tower of London misst die mentalen Planungs- und Problemlösefähigkeiten.

Res Dev Disabil 2021; 110: 103862

\section{CO-OP}

Der Cognitive Orientation to daily Occupational Performance Approach (CO-OP) ist eine betätigungszentrierte, zielorientierte Intervention für Kinder. Mittels metakognitiver Strategien fördert der Ansatz Veränderungen der Betätigungsperformanz. Seine Problemlösestrategie „Ziel-Plan-TuCheck“ regt den Einsatz von Gedächtnis, Aufmerksamkeit und mentaler Planung an. Res Dev Disabil 2021; 110: 103862 in die Therapie ihrer Kinder einzubeziehen.

Res Dev Disabil 2021; 110: 103862 